\title{
EDITORIAL
}

\section{The Cerebellum: Not Just an Anatomical Structure}

\author{
*Adekomi AD. ${ }^{1}$, Adewole OS. ${ }^{2}$, Tijani AA. ${ }^{3}$, Shallie P. ${ }^{4}$
}

In human, the cerebellum occupies about ten percent of the total skull. It contains numerous cells one of which is the Purkinje cell. Traditionally, the cerebellum is made up of four important types of neuronal cells namely Golgi cells, granules cells, Purkinje cells, and; stellate cells (basket cells). However, with the rapid advances in medical sciences, it is now known that the cerebellum contains many other cells, these includes; candelabrum cells, Lugaro cells, and unipolar brush cells. The fact that these neuronal cells are usually not cited in the standard basic expression of the microscopic anatomy of the cerebellum, or even in standard anatomy, physiology, or histology literatures, indicate that our knowledge of the existence of these cells is fractional (1).

Recent microscopic study by Tang et al. (2016) (2) has shown that the cerebellum contains the significant number of neuronal cells and synapses compared with any other structure within the central nervous system. The functions of these wide arrays of neurons are determined by a set of biological stimulus (i.e. excitatory or inhibitory inputs). According to Heshmat (2016) (3), excitatory inputs are generated by many mossy fibers which developed from the pontine nuclei and climbing fibers originating from the inferior olivary nucleus. On the other hand, large inhibitory inputs arise from the Purkinje cells situated in the cerebellar cortex. Observations made from the neurobiology of the cerebellum showed that there is need for integration between excitatory and inhibitory inputs so as to develop the output signal required for the functional integrity of the cerebellum. The inhibitory input from a single cerebellar nucleus is usually opposed by depolarizing potentials in the recipient cerebellar nuclei cells due to spontaneous depolarization activity in the presence of such an inhibitory input (3).

Evidences from cognitive studies further suggest that cerebellar pathology may be associated with alterations mainly in mental function, instead of motor processes. These pools of evidences continue to attract a sizeable number of researches into the neuroanatomy, neurobiology and neurobehavioral role of the cerebellum and the theories associated with its functions.

\section{REFERENCES}

1. Schilling K, Oberdick J, Rossi F, Baader SL (2008): Besides Purkinje cells and granule neurons: an appraisal of the cell biology of the interneurons of the cerebellar cortex. Histochem Cell Biol 130:601-615

2. Tang T, Suh CY, Blenkinsop TA, Lang EJ (2016): Synchrony is key: Complex spike inhibition of the deep cerebellar nuclei. Cerebellum 15(1):10-13

3. Heshmat SWH (2016). Cerebellar Nuclei and Connections in Man. Anatomy Physiol Biochem Int J. 1(1): 001-008.

\footnotetext{
*Correspondence author:Adedayo Damilare Adekomi ${ }^{\dagger+}$ Ph.D, E-mail: adedayo.adekomi@ uniosun.edu.ng

${ }^{1}$ Department of Anatomy, Osun State University, Osogbo, Nigeria.

${ }^{2}$ Department of Anatomy and Cell Biology, Obafemi Awolowo University, Ile-Ife, Nigeria.

${ }^{3}$ Department of Anatomy, Ekiti State University, Ado-Ekiti, Nigeria.

${ }^{4}$ Department of Anatomy, Olabisi Onabanjo University, Ago-Iwoye, Nigeria.
}

Research Journal of Health Sciences subscribed to terms and conditions of Open Access publication. Articles are distributed under the terms of Creative Commons Licence (CC BY-NC-ND 4.0). (http://creativecommons.org/licences/by-nc-nd/4.0). 


\title{
ÉDITORIAL
}

\section{Le cervelet: pas seulement une structure anatomique}

\author{
*Adekomi AD. ${ }^{1}$, Adewole OS. ${ }^{2}$, Tijani AA. ${ }^{3}$, Shallie P. ${ }^{4}$
}

Chez l'homme, le cervelet occupe environ $10 \%$ du crâne total. Il contient de nombreuses cellules dont l'une est la cellule de Purkinje. Traditionnellement, le cervelet est composé de quatre types importants de cellules neuronales, à savoir les cellules de Golgi, les cellules de granules, les cellules de Purkinje et; Cellules étoilées (cellules de panier). Cependant, avec les progrès rapides dans les sciences médicales, on sait maintenant que le cervelet contient beaucoup d'autres cellules, cela inclut; Les cellules candélabres, les cellules Lugaro et les cellules brosses unipolaires. Le fait que ces cellules neuronales ne soient habituellement pas citées dans l'expression de base standard de l'anatomie microscopique du cervelet, ou même dans les littératures normalisées d'anatomie, de physiologie ou d'histologie, indiquent que notre connaissance de l'existence de ces cellules est fractionnaire (1).

Une étude microscopique récente de Tang et al. (2016) (2) a montré que le cervelet contient le nombre significatif de cellules neuronales et de synapses par rapport à toute autre structure dans le système nerveux central. Les fonctions de ces réseaux étendus de neurones sont déterminées par un ensemble de stimulus biologiques (c'est-à-dire des entrées excitatrices ou inhibitrices). Selon Heshmat (2016) (3), les intrants excitateurs sont générés par de nombreuses fibres moussues qui se sont développées à partir des noyaux ponctuels et des fibres d'escalade provenant du noyau olivaire inférieur. D'autre part, des intrants inhibiteurs importants proviennent des cellules de Purkinje situées dans le cortex cérébelleux. Les observations faites à partir de la neurobiologie du cervelet montrent qu'il est nécessaire d'intégrer les entrées excitatrices et inhibitrices pour développer le signal de sortie nécessaire à l'intégrité fonctionnelle du cervelet. L'apport inhibiteur provenant d'un seul noyau cérébelleux est habituellement opposé par des potentiels dépolarisants dans les cellules nucléaires cérébelleuses receveuses en raison de l'activité spontanée de dépolarisation en présence d'une telle entrée inhibitrice (3).

Les preuves des études cognitives suggèrent en outre que la pathologie cérébelleuse peut être associée à des altérations principalement dans la fonction mentale, au lieu des processus moteurs. Ces pools de preuves continuent d'attirer un nombre considérable de recherches sur la neuroanatomie, la neurobiologie et le rôle neurocomportemental du cervelet et les théories associées à ses fonctions.

\section{REFERENCES}

1. Schilling K, Oberdick J, Rossi F, Baader SL (2008): Besides Purkinje cells and granule neurons: an appraisal of the cell biology of the interneurons of the cerebellar cortex. Histochem Cell Biol 130:601-615

2. Tang T, Suh CY, Blenkinsop TA, Lang EJ (2016): Synchrony is key: Complex spike inhibition of the deep cerebellar nuclei. Cerebellum 15(1):10-13

3. Heshmat SWH (2016). Cerebellar Nuclei and Connections in Man. Anatomy Physiol Biochem Int J. 1(1): 001-008.

\footnotetext{
*Correspondence author: Adedayo Damilare Adekomi ${ }^{* \dagger}$ Ph.D, E-mail: adedayo.adekomi@ uniosun.edu.ng

${ }^{1}$ Department of Anatomy, Osun State University, Osogbo, Nigeria.

${ }^{2}$ Department of Anatomy and Cell Biology, Obafemi Awolowo University, Ile-Ife, Nigeria.

${ }^{3}$ Department of Anatomy, Ekiti State University, Ado-Ekiti, Nigeria.

${ }^{4}$ Department of Anatomy, Olabisi Onabanjo University, Ago-Iwoye, Nigeria.
} 